\section{Stenting versus Thrombendarteriektomie bei asymptomatischer hochgradiger Karotisstenose}

\author{
Zur Behandlung einer hochgradigen, aber asymptomatischen Karotisstenose \\ liefert die Studie ACT I neue Daten, aber wenig Neues.
}

$\mathrm{n}$ die prospektive, multizentrisch in den USA durchgeführte Studie ACT I wurden 1.453 Patienten mit hochgradiger Karotisstenose (70-99\% nach NASCET) eingeschlossen. Die Stenose war asymptomatisch, was als Freiheit von ischämischen zerebrovaskulären Ereignissen in den letzten 180 Tagen vor der Randomisierung definiert wurde.

Die Probanden wurden im Verhältnis 3:1 in eine Stent- und eine OP-Gruppe verteilt. Alle Patienten erhielten als Basismedikation ASS (325 mg/d) ab mindestens drei Tage vor dem Eingriff bzw. der Intervention. Die Patienten mit geplantem Stenting bekamen zusätzlich für einen Monat Clopidogrel. In der operativ behandelten Gruppe oblag die Wahl von OP-Technik und Anästhesie dem Operateur. Die Probanden mit stentgeschützter Angioplastie erhielten einen Nitinolstent unter Verwendung eines Embolie-Protektionssystems. Als primärer Endpunkt wurde eine Kombination aus Tod, Schlaganfall jedweder Lokalisation und Myokardinfarkt innerhalb der ersten 30 Tage nach dem Eingriff sowie ein ipsilateraler Schlaganfall zwischen Tag 31 und 365 definiert. Zudem wurden eine Reihe sekundärer Endpunkte, u. a. die Rate an ipsilateralen Schlaganfällen nach fünf Jahren, festgelegt. Die Studie war auf Nichtunterlegenheit des Stentings ausgelegt.

Periprozedural, d.h. in den ersten 30 Tagen, kam es in der operativen Gruppe bei 2,6\% zu primären Endpunktereignissen, in der Stentgruppe bei 3,3\% (p= $0,6)$. Dabei zeigte sich ein nicht signifikanter Trend zu mehr Schlaganfällen in der Angioplastiegruppe (2,8\%) im Vergleich zu den operierten Patienten (1,4\%; $\mathrm{p}=0,23)$, der durch das häufigere Auftreten von Myokardinfarkten in der OPGruppe $(0,9$ vs. $0,5 \%$; $p=0,41)$ kompensiert wurde. Die Rate von Schlaganfällen, die nicht mit dem Eingriff assoziiert waren, war innerhalb einer fünfährigen Nachbeobachtung in beiden Gruppen ebenfalls gleich hoch $(2,2 \%=0,4 \% / \mathrm{Jahr}$ nach der Angioplastie versus 2,7\% = $0,5 \% /$ Jahr nach einer Thrombendarterieektomie). Damit wurde die Nichtunterlegenheit der stentgestützten Angioplastie gegenüber der Thrombendarterieektomie postuliert.

Rosenfield K et al. Randomized Trial of Stent versus Surgery for Asymptomatic Carotid Stenosis. N Engl J Med. 2016;374:1011-31

\section{Kommentar}

ACT I ist die erste prospektive Studie, die fokussiert die Behandlung asymptomatischer hochgradiger Karotisstenosen mittels stentgeschützter Angioplastie im Vergleich zur Thrombendarteriektomie untersucht hat. Dabei konnte - entsprechend geschulte Interventionalisten vorausgesetzt - die Nichtunterlegenheit des Stentings bezüglich harter periprozeduraler Endpunkte (Tod, Schlaganfall, Myokardinfarkt) demonstriert werden, wenngleich ein Trend unterlegen zu sein. zu einer höheren Rate an Schlaganfällen in den ersten 30 Tagen erkennbar war. Die 2010 publizierte CREST-Studie, die symptomatische und asymptomatische Patienten einschloss, hatte bezüglich des primären Endpunkts nahezu identische Ergebnisse erbracht, so dass ACT I nicht wirklich neue Erkenntnisse liefert.

Viel entscheidender ist jedoch die Frage, inwieweit bei asymptomatischen Karotisstenosen heute überhaupt noch eine interventionelle oder operative Behandlung indiziert ist. Beobachtungsstudien der letzten 15 Jahre zeigen unter optimierter Medikation (ASS, Blutdrucksenker, Statine), Diät und Lebensstilmodifikation eine stete Abnahme des Schlaganfallrisikos. Bedauerlicherweise wurde die SPACE-2-Studie, die neben Operation und Stenting auch einen ausschließlich medikamentösen Behandlungsarm enthielt, 2015 aufgrund der äußerst schleppenden Rekrutierung eingestellt. Antwort auf diese Frage wird damit wohl erst die in den USA laufende CREST2-Studie mit ähnlichem Design wie SPACE-2 liefern, deren Ergebnisse jedoch nicht vor 2020 zu erwarten sind.

\section{Dr. med. Sven Lerch}

Abteilung Neurologie,

Bundeswehrkrankenhaus Berlin

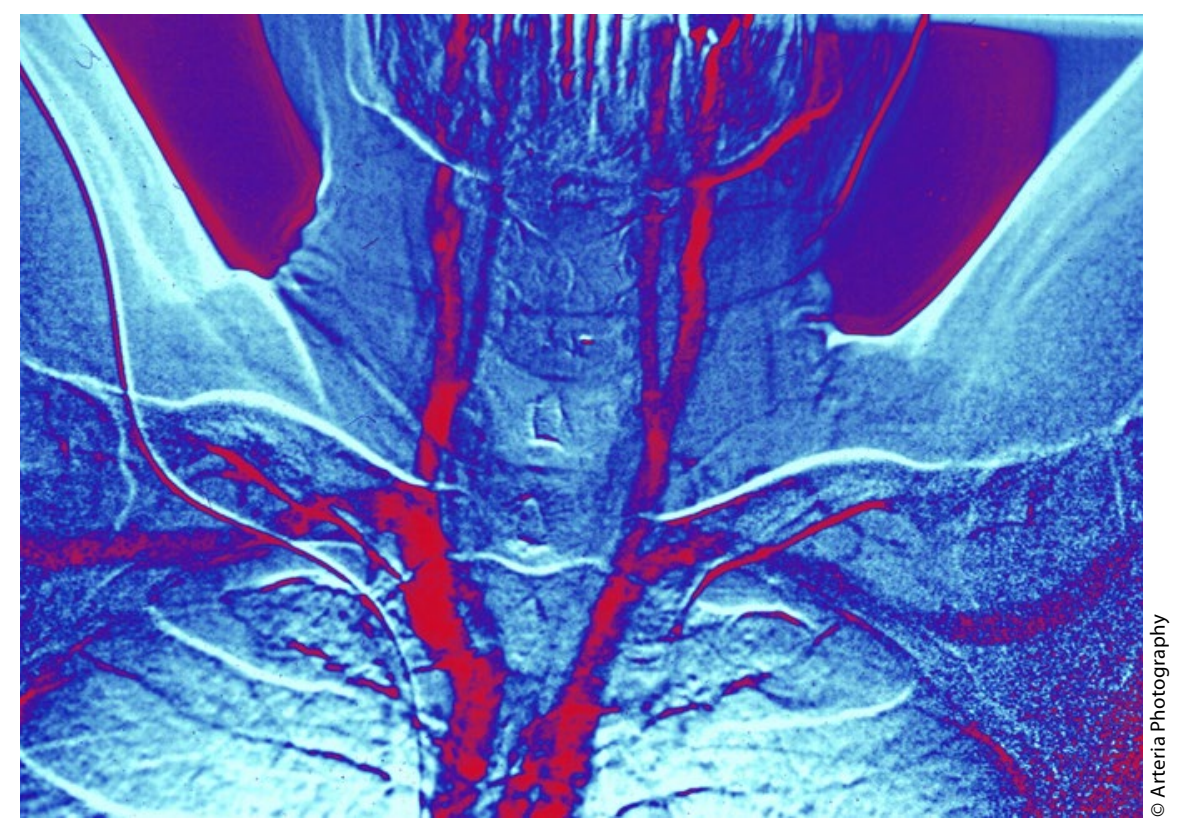

Bei asymptomatischen Karotisstenosen scheint das Stenting einer Operation nicht 